\title{
Relationship between maternal gestational hypertension and home blood pressure in 7-year-old children and their mothers: Tohoku Study of Child Development
}

\author{
Miki Hosaka ${ }^{1}$, Kei Asayama ${ }^{1,2}$, Jan A Staessen ${ }^{3,4}$, Nozomi Tatsuta ${ }^{5,6}$, Michihiro Satoh ${ }^{7}$, Masahiro Kikuya ${ }^{8}$, \\ Takayoshi Ohkubo ${ }^{2}$, Hiroshi Satoh ${ }^{9}$, Yutaka Imai $^{1}$ and Kunihiko Nakai ${ }^{5,6}$
}

Women who had hypertensive disorders in pregnancy have an increased risk of cardiovascular diseases in later life. No studies, however, have investigated whether maternal hypertensive disorders in pregnancy affect self-measured blood pressure at home (HBP) in mothers and their children. We evaluated the association between maternal hypertension during pregnancy and HBP based on the prospective Tohoku Study of Child Development birth cohort study, which was performed in two areas in Japan. We included children in a singleton birth at term (36-42 weeks of gestation) with a birth weight of $>2400 \mathrm{~g}$. We collected prenatal care data from the medical charts. Because only two mothers experienced preeclampsia, we defined gestational hypertension (GH) as a hypertensive disorder in pregnancy. Seven years after birth, mothers and their children measured their HBP in the morning for 2 weeks. Of 813 eligible mothers, 28 (3.4\%) experienced GH, and those were of a similar age compared with 785 non-GH mothers (37.3 vs. 38.0 years; $P=0.41)$. Women with GH had higher body mass index (BMI) $\left(23.8 v s .21 .4 \mathrm{~kg} \mathrm{~m}^{-2}\right.$; $P=0.01)$ and elevated HBP $(120.3 / 76.8$ vs. $110.4 / 68.6 \mathrm{~mm} \mathrm{Hg} ; \boldsymbol{P}<0.0002) 7$ years after delivery. However, HBP was similar in children with and without GH mothers $(93.5 / 55.9$ vs. $94.1 / 56.1 \mathrm{~mm} \mathrm{Hg}, P>0.38)$. These results were confirmatory in casecontrol $(1: 2)$ analyses with matching by maternal age, maternal BMI before pregnancy, survey area and parity. In conclusion, maternal GH did not affect HBP in offspring but strongly affected maternal HBP even 7 years after birth. Hypertension Research (2015) 38, 776-782; doi:10.1038/hr.2015.63; published online 14 May 2015

Keywords: child; gestational hypertension; home blood pressure; prospective birth cohort study; self-measurement

\section{INTRODUCTION}

Hypertension is a major cardiovascular risk factor in adults, ${ }^{1-4}$ and the risk of developing high blood pressure (BP) during adolescence can be predicted based on BP in childhood. ${ }^{5}$ Children with high BP are at an increased risk of sustained hypertension as well as metabolic syndrome in adulthood. ${ }^{6}$ Meanwhile, self-measurement of BP at home (HBP), which has been recognized as a useful tool for the accurate diagnosis and treatment of hypertension in adults, ${ }^{7,8}$ would be superior to the conventional BP (CBP) measurements in children as well, regarding the long-term reproducibility and the prognostic significance of the presence of subclinical end-organ damage. ${ }^{9,10}$ Nevertheless, current data on HBP in children are limited.
Pregnancy-induced hypertension (PIH), diagnosed by increased $\mathrm{BP}$ with or without proteinuria (preeclampsia or gestational hypertension (GH)), is a common complication in pregnancy. ${ }^{11,12}$ PIH occurs in 7-12\% of all singleton pregnancies worldwide. ${ }^{13}$ Even in children and adolescents, the offspring from preeclampsia or $\mathrm{GH}$ mothers reportedly have higher ambulatory BP or CBP. ${ }^{14-18}$ To the best of our knowledge, however, no studies have investigated whether maternal hypertension during pregnancy affects self-measured HBP in mothers and their offspring. We aimed to investigate the association between maternal hypertensive disorders during pregnancy and both $\mathrm{HBP}$ and $\mathrm{CBP}$ in young children and mothers after 7 years of the delivery.

${ }^{1}$ Department of Planning for Drug Development and Clinical Evaluation, Tohoku University Graduate School of Pharmaceutical Sciences, Sendai, Japan; ${ }^{2}$ Department of Hygiene and Public health, Teikyo University School of Medicine, Tokyo, Japan. ${ }^{3}$ Studies Coordinating Centre, Research Unit Hypertension and Cardiovascular Epidemiology, KU Leuven Department of Cardiovascular Sciences, University of Leuven, Leuven, Belgium; ${ }^{4}$ VitaK Research and Development, Maastricht University, Maastricht, The Netherlands; ${ }^{5}$ Department of Environmental Health Sciences, Tohoku University Graduate School of Medicine, Sendai, Japan; ${ }^{6}$ Department of Development and Environmental Medicine, Tohoku University Graduate School of Medicine, Sendai, Japan; ${ }^{7}$ Department of Pharmacy, Tohoku University Hospital, Sendai, Japan; ${ }^{8}$ Department of Preventive Medicine and Epidemiology, Tohoku Medical Megabank Organization, Tohoku University, Sendai, Japan and ${ }^{9}$ Professor Emeritus, Tohoku University, Sendai, Japan 


\section{METHODS}

\section{Participants}

The Tohoku Study of Child Development, conducted in the northeastern region of Japan, is a prospective birth cohort study investigating the health and development of children. Details of the Tohoku Study of Child Development have been described previously. ${ }^{19,20}$ From January 2001 to September 2005, we enrolled 1566 pregnant women. Mothers were eligible if they had no severe diseases such as thyroid dysfunction, hepatitis, immune deficiency, malignant tumors or mental diseases; if they had not undergone in vitro fertilization; and if they spoke Japanese as their native language. A total of 1348 babies fulfilled the inclusion criteria as shown in Figure 1: the absence of congenital anomalies or severe diseases and a singleton birth at term (36-42 weeks of gestation) with a birth weight of $>2400 \mathrm{~g}$. Of those, 953 children at 7 years of age and their mothers were surveyed in a quiet examination room by 31 March 2013. We excluded 138 children from the current analysis because either their BP was unavailable $(n=71)$, their HBP in the morning was measured for $<3$ days $(n=37)$, the mothers were taking antihypertensive medication during the survey $(n=3)$, or the required characteristics or outcomes at the 7-year survey for analysis were not fully available $(n=27)$. We further excluded mothers who experienced preeclampsia because of the limited number $(n=2)$. Therefore, the study included 813 mother-offspring pairs (Figure 1). There were no significant differences in gestational age, birth weight and height, maternal age, prevalence of sex or parity between the 813 analyzed children and the 138 who were not included $(P>0.12)$. We obtained written informed consent from the mothers, and the Ethics Committee of Tohoku University Graduate School of Medicine approved the study. ${ }^{19,20}$

\section{Information on mothers during pregnancy}

Characteristics of mothers during pregnancy were obtained from medical records during the perinatal period. A urinary protein dip-stick test was performed at every 2- to 4 -week visit to the hospital. CBP during pregnancy was also measured at every visit after the participant rested for a few minutes in a sitting position. The median number of visits to the hospital during pregnancy was 12 (interquartile range, 11-13). Information on smoking and drinking habits during pregnancy, parity and education was collected by selfadministered questionnaires at enrollment or 4 days after the delivery.

Hypertension during pregnancy was defined as CBP $\geqslant 140 \mathrm{~mm} \mathrm{Hg}$ systolic and/or BP $\geqslant 90 \mathrm{~mm} \mathrm{Hg}$ diastolic. Proteinuria was diagnosed if the protein reading on dip-stick testing was $1+$ or more. ${ }^{21}$ Preeclampsia was defined as de novo hypertension on at least two occasions after 20 weeks of gestation accompanied by proteinuria. ${ }^{11,12} \mathrm{GH}$ was defined as de novo hypertension on at least two occasions after 20 weeks of gestation, but without proteinuria. ${ }^{11,12}$ We found no participants with superimposed preeclampsia, which was defined as fulfilling preeclampsia criteria along with hypertension or proteinuria on at least one occasion before 20 weeks of gestation. ${ }^{11,12}$ We therefore defined PIH mothers as those with preeclampsia and GH.

\section{BP measurement and other information on the 7-year survey}

On the 7-year survey, the CBP and HBP in mothers and children were measured using an OMRON HEM-7080IC (Omron Healthcare, Kyoto, Japan), a device based on the cuff-oscillometric method that generates a digital display of both systolic and diastolic BP values. The device used in the present study is equivalent to the OMRON HEM-705IT and has been validated. ${ }^{20,22} \mathrm{We}$ downloaded the BP readings directly to a computer from the device without any measurement bias. ${ }^{20}$ Small, standard and large cuffs had inflatable bladders of $16 \times 9 \mathrm{~cm}, 22 \times 12 \mathrm{~cm}$ and $30 \times 15 \mathrm{~cm}$, respectively, and were used in mothers and offspring depending on upper-arm circumference (under $22 \mathrm{~cm}$, $22-32 \mathrm{~cm}$ and over $32 \mathrm{~cm}$, respectively).

The CBP was measured by trained survey staff; the measurements were performed once on mothers and children in a seated position after resting for at least $2 \mathrm{~min}$. The study participants were also instructed and trained how to measure their HBP, and they were asked to take their own measurements for 2 weeks. Their HBP was measured once in the morning before breakfast, in the sitting position, within $1 \mathrm{~h}$ after awaking and after 2 min or more of rest. HBP in each child was measured with the mother's assistance. Although many participants measured their HBP twice or more per occasion, we used the first value from each measurement in our analysis to exclude individual selection bias, and the mean of all first measurements were used as HBP. The initial HBP was defined as first HBP measurement from the first day for each participant. Maternal conventional hypertension was defined as CBP $\geqslant 140 \mathrm{~mm} \mathrm{Hg}$ for systolic and/or $\mathrm{BP} \geqslant 90 \mathrm{~mm} \mathrm{Hg}$ for diastolic, and maternal home hypertension as $\mathrm{HBP} \geqslant 135 / \geqslant 85 \mathrm{~mm} \mathrm{Hg}$.

The heights and weights of the children at 7 years of age were measured using a KS-502Gp automatic analyzer (Kansai Seiki, Kusatsu, Japan), whereas the participants wore light indoor clothes and no shoes. We calculated the body mass index (BMI) using the following formula: weight $(\mathrm{kg}) /$ height $(\mathrm{m})^{2}$.

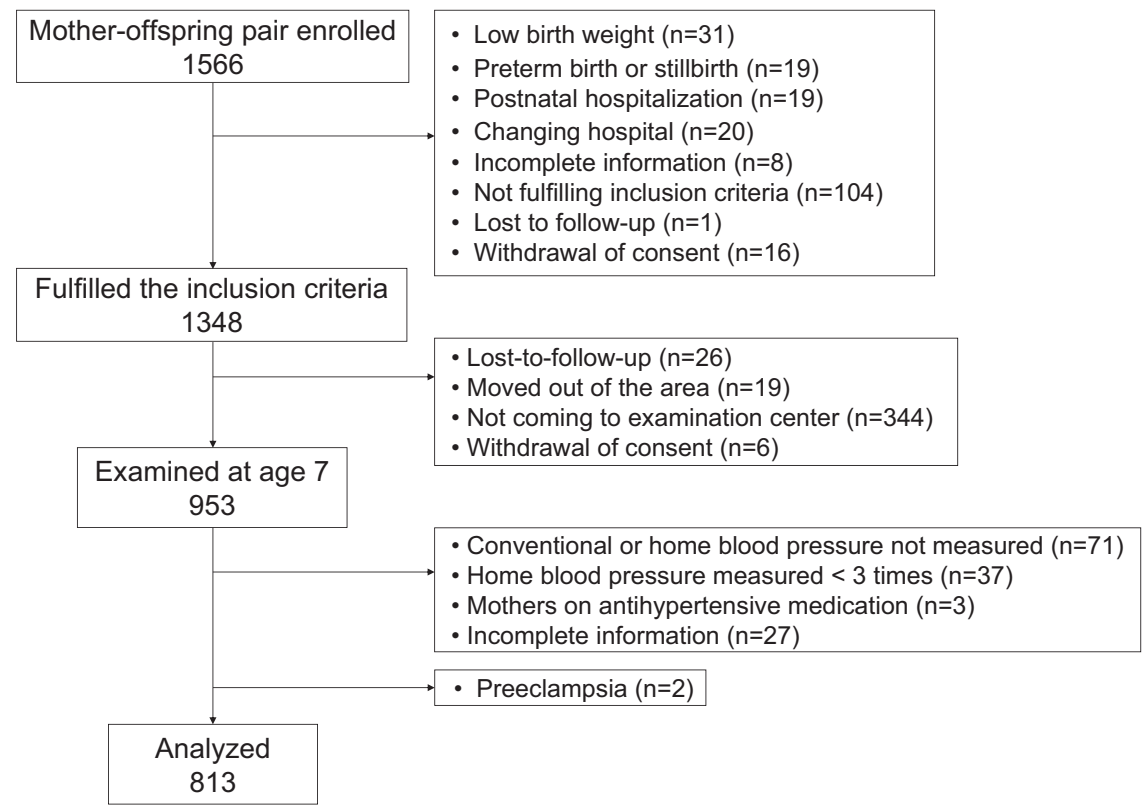

Figure 1 Flowchart of the study population. 


\section{Statistical analyses}

We used SAS software (version 9.3, SAS Institute Cary, NC, USA) for database management and statistical analysis. All data are presented as the mean \pm s.d. unless otherwise stated. Statistical significance was established at $P<0.05$.

The variables for the characteristics of the study participants and the children's outcomes at the 7-year survey were compared using Fisher's exact test or Student's $t$-test appropriately. For the comparison of CBP and HBP between the two groups, we used analysis of covariance adjusted by the following factors according to the previous studies: ${ }^{15,20,23}$ for the children, sex, birth weight, age at 7-year survey, BMI at 7-year survey, parental education status, maternal age at delivery, parity, maternal smoking during pregnancy, maternal BMI before pregnancy, cesarean section and gestational week; for the mothers, age and BMI at 7 -year survey, education ( $\geqslant 13$ or $<13$ years), parity, family history of hypertension, and current drinking and smoking status. BP variability, represented by s.d. and coefficient of variation, was also compared between the two groups.

Along with the whole-population analysis, we conducted matched casecontrol analysis to equalize baseline characteristics in the participants. Each mother diagnosed with PIH was paired with two controls matched for maternal age at delivery (an age range of \pm 6 years with respect to each case), maternal BMI before pregnancy $\left( \pm 6 \mathrm{~kg} \mathrm{~m}^{-2}\right)$, parity and residence-factors thought to have great influence on maternal PIH; the remaining controls were discarded.

\section{RESULTS}

Of the 813 children at age 7 (mean $84.7 \pm 1.7$ months) and their mothers included in this study, 28 mothers $(3.4 \%)$ were diagnosed with GH. On the 7-year survey, 42 (5.2\%) and 46 (5.7\%) mothers had conventional and home hypertension, respectively. Table 1 shows the characteristics of the GH, non-GH and matched non-GH groups of mothers and children. Maternal BMI before pregnancy and on the 7 -year survey in the GH group were $23.8 \pm 4.7$ and $23.8 \pm 4.9 \mathrm{~kg} \mathrm{~m}^{-2}$, respectively, which were significantly higher than those in the non-GH group, $21.0 \pm 2.8$ and $21.4 \pm 3.0 \mathrm{~kg} \mathrm{~m}^{-2}$, respectively $(P=0.004 / 0.01)$. The rate of family history of hypertension was also higher in mothers with GH than those with non-GH $(67.9 \%$ vs. $39.0 \%, P=0.003)$. No significant differences were observed in other variables $(P>0.12)$. After matching with maternal age, maternal BMI, parity and residence, we found no significant differences in their characteristics between 28 matched cases and 56 controls in the case-matching analysis $(P \geqslant 0.07)$ except for drinking during pregnancy $(P=0.03)$.

In our population, the 95th percentile of children's conventional and home BP were $109 / 72$ and $106 / 65 \mathrm{~mm} \mathrm{Hg}$, respectively. After adjusting for the confounding factors, there were no significant differences $(P \geqslant 0.38)$ in children's BP between the non-GH group $(93.1 \pm 9.2 / 57.4 \pm 9.1 \mathrm{~mm} \mathrm{Hg}$ for $\mathrm{CBP}$ and

Table 1 Characteristics of mothers and children by GH categories

\begin{tabular}{|c|c|c|c|}
\hline Variables & Non-GH $(\mathrm{n}=785)$ & $G H(n=28)$ & Matched non-GH $(\mathrm{n}=56)$ \\
\hline \multicolumn{4}{|l|}{ Mothers } \\
\hline Age at delivery, years & $30.9 \pm 4.5$ & $30.2 \pm 5.0$ & $30.4 \pm 4.7$ \\
\hline Age at 7-year survey, years & $38.0 \pm 4.5$ & $37.3 \pm 5.0$ & $37.5 \pm 4.7$ \\
\hline BMI before pregnancy, $\mathrm{kg} \mathrm{m}^{-2}$ & $21.0 \pm 2.8$ & $23.8 \pm 4.7$ & $23.4 \pm 4.1$ \\
\hline BMI at 7-year survey, $\mathrm{kg} \mathrm{m}^{-2}$ & $21.4 \pm 3.0$ & $23.8 \pm 4.9$ & $23.1 \pm 3.8$ \\
\hline Smoking during pregnancy, $n(\%)$ & $76(9.7)$ & $2(7.1)$ & $6(10.7)$ \\
\hline Smoking at 7-year survey, $n(\%)$ & $115(14.7)$ & $2(7.1)$ & $8(14.3)$ \\
\hline Drinking during pregnancy, $n(\%)$ & $190(24.3)$ & $3(10.7)$ & $18(32.7)$ \\
\hline Drinking at 7-year survey, $n(\%)$ & $330(42.0)$ & $10(35.7)$ & $22(39.3)$ \\
\hline No previous pregnancies, $n(\%)$ & $374(47.6)$ & $16(57.1)$ & $32(57.1)$ \\
\hline Education $\geqslant 13$ years, $n(\%)$ & $462(59.5)$ & $21(75.0)$ & $38(67.9)$ \\
\hline Family history of hypertension, $n(\%)$ & $306(39.0)$ & 19 (67.9) & $26(46.4)$ \\
\hline Cesarean section, $n(\%)$ & $133(16.9)$ & 5 (17.9) & $11(19.6)$ \\
\hline \multicolumn{4}{|l|}{ Heart rate } \\
\hline Conventional, beats $\min ^{-1}$ & $68.0 \pm 9.3$ & $67.7 \pm 7.3$ & $68.8 \pm 9.3$ \\
\hline Home, beats $\min ^{-1}$ & $69.2 \pm 7.5$ & $69.3 \pm 6.1$ & $69.6 \pm 7.0$ \\
\hline \multicolumn{4}{|l|}{ Children } \\
\hline Girls, $n(\%)$ & $373(47.5)$ & $14(50.0)$ & $25(44.6)$ \\
\hline Gestational age, months & $39.6 \pm 1.2$ & $39.7 \pm 1.3$ & $39.9 \pm 1.1$ \\
\hline Age at 7-year survey, months old & $84.7 \pm 1.7$ & $84.4 \pm 1.6$ & $84.9 \pm 1.6$ \\
\hline Birth weight, g & $3121.2 \pm 357.6$ & $3115.6 \pm 399.5$ & $3180.1 \pm 344.8$ \\
\hline Weight at 7-year survey, kg & $23.3 \pm 3.5$ & $23.4 \pm 2.7$ & $24.1 \pm 4.0$ \\
\hline Height at 7-year survey, cm & $120.1 \pm 4.8$ & $119.0 \pm 4.3$ & $120.6 \pm 4.7$ \\
\hline $\mathrm{BMI}$ at 7-year survey, $\mathrm{kg} \mathrm{m}^{-2}$ & $16.1 \pm 1.6$ & $16.5 \pm 1.4$ & $16.5 \pm 1.9$ \\
\hline \multicolumn{4}{|l|}{ Heart rate } \\
\hline Conventional, beats $\min ^{-1}$ & $79.9 \pm 10.4$ & $79.2 \pm 8.8$ & $78.9 \pm 10.7$ \\
\hline Home, beats $\min ^{-1}$ & $84.1 \pm 7.6$ & $85.6 \pm 7.7$ & $84.5 \pm 7.4$ \\
\hline
\end{tabular}

Abbreviations: BMI, body mass index; GH, gestational hypertension. Numbers are shown as mean \pm s.d. or $\%$ in parentheses, appropriately. Drinking during pregnancy in non-GH and matched nonGH mothers, and education status in non-GH mothers were unavailable in 2,1 and 9 , respectively. All of the Fisher's exact test and student's $t$-test statistic $P$-values for differences between non$\mathrm{GH}$ and $\mathrm{GH}$ were not significant $(P>0.12)$ except for maternal BMI before pregnancy and at 7-year survey, and family history of hypertension $(P<0.014)$.

Matched non-GH: each case who was diagnosed GH was paired to two controls matched for maternal age at delivery, maternal BMI before pregnancy, parity and residence. There were no significant differences on characteristics between GH and matched non-GH $(P \geqslant 0.07)$ except for drinking during pregnancy $(P=0.03)$. 
$94.1 \pm 6.9 / 56.1 \pm 5.7 \mathrm{~mm} \mathrm{Hg}$ for HBP) and the GH group (90.0 $\pm 6.4-$ $/ 56.7 \pm 7.0 \mathrm{~mm} \mathrm{Hg}$ for $\mathrm{CBP}$ and $93.5 \pm 5.7 / 55.9 \pm 4.1 \mathrm{~mm} \mathrm{Hg}$ for HBP) except for systolic CBP $(P=0.04)$. The mean $(95 \%$ confidence intervals) differences between the two groups were $3.6(0.2-7.0) / 0.8$ $(-2.6-4.2) \mathrm{mmHg}$ for CBP and $1.1(-1.3-3.5) / 0.6(-1.5-2.7) \mathrm{mmHg}$ for HBP (Figure 2). Maternal HBP as well as CBP in the GH group $(123.7 \pm 20.3 / 79.8 \pm 12.8 \mathrm{~mm} \mathrm{Hg}$ for CBP and $120.3 \pm 12.7-$ $176.8 \pm 10.3 \mathrm{~mm} \mathrm{Hg}$ for $\mathrm{HBP}$ ) was significantly higher than in the non-GH group $(109.5 \pm 12.6 / 70.5 \pm 9.6 \mathrm{~mm} \mathrm{Hg}$ for $\mathrm{CBP}$ and $110.4 \pm 10.9 / 68.6 \pm 8.3 \mathrm{~mm} \mathrm{Hg}$ for HBP); the mean (95\% confidence intervals) differences were 10.6 (6.3-15.0)/6.9 (3.5-10.3) $\mathrm{mmHg}$ for CBP and 6.8 (3.2-10.5)/6.1 (3.3-9.0) $\mathrm{mmHg}$ for HBP after applying multivariable adjustment for possible confounders $(P<0.0002)$ (Figure 3$)$. The results were essentially similar when the initial HBP instead of the average HBP was used (Table 2). No significant differences in the s.d. and coefficient of variation of systolic/diastolic HBP were observed between the two groups of children $(P>0.22)$ and mothers $(P>0.07)$.

Figure 4 shows the 1:2 matched case-control analysis. Although there were no significant differences between the matched non-GH and GH groups in terms of their characteristics (Table 1), maternal HBP in the GH group was significantly higher than in the matched non-GH group even after adjustment $(P<0.002)$. However, children's HBP was statistically at the same level between the two groups (Figure $4 ; P>0.41$ ). In children, only systolic CBP was significantly higher in the non-GH group than in the GH group $(3.9(95 \%$ confidence intervals: $0.8-6.9) \mathrm{mmHg}, P=0.01$ ).

Results were confirmed when we included two preeclampsia mothers and their children into the study population as well as the evaluated BP levels in $30 \mathrm{PIH}$ mothers and their children (Table 3).
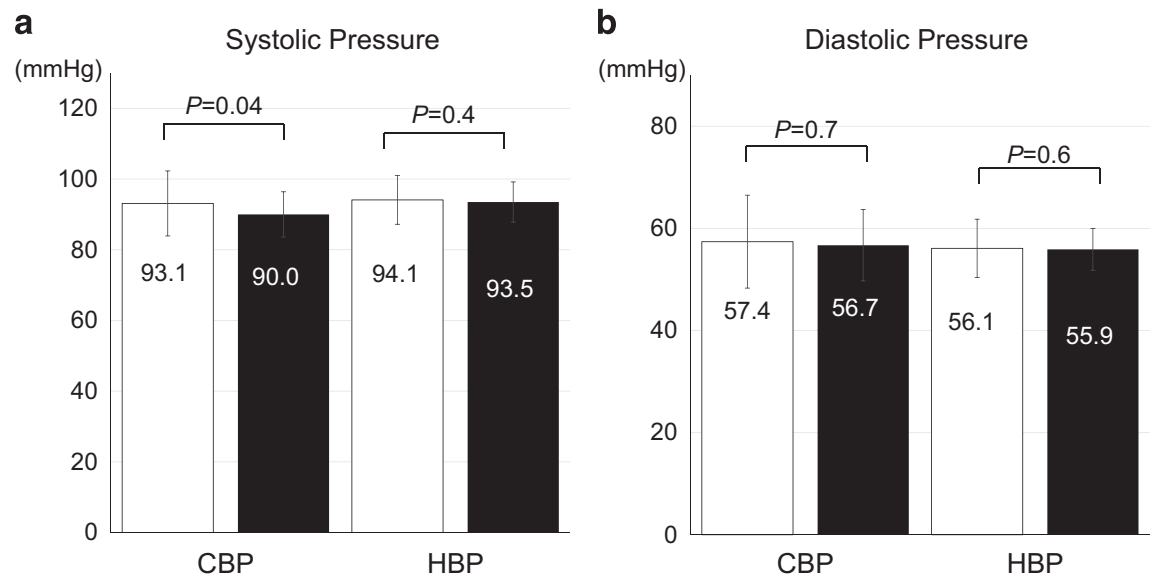

Figure 2 Children's CBP and HBP in the non-GH group (open bar; $n=785$ ) and the GH group (solid bar; $n=28$ ). Panel a, systolic blood pressure; panel $\mathbf{b}$, diastolic blood pressure. CBP and HBP were analyzed using analysis of variance adjusted for sex, birth weight, age at 7-year survey, children's body mass index at 7-year survey, parental education status, maternal age at delivery, parity, maternal smoking during pregnancy, maternal body mass index before pregnancy, cesarean section and gestational week. Error bars denote s.d. P-values express the differences between GH and non-GH after adjusting for sex, birth weight, age at 7-year survey, children's body mass index at 7-year survey, parental education status, maternal age at delivery, parity, maternal smoking during pregnancy, maternal body mass index before pregnancy, cesarean section and gestational week. GH, gestational hypertension; CBP, conventional blood pressure; HBP, home blood pressure.
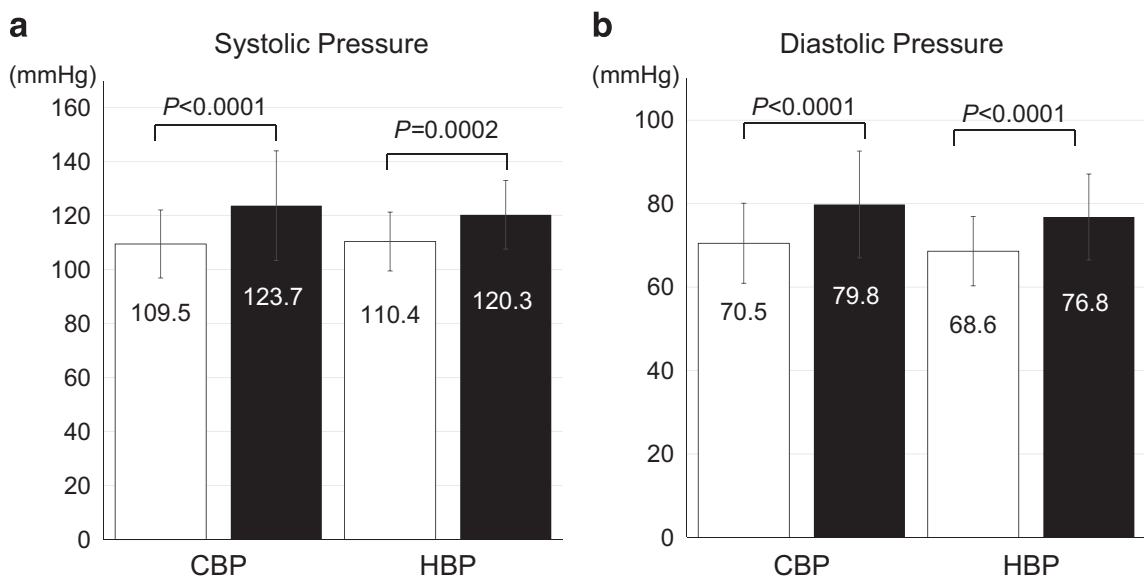

Figure 3 Maternal CBP and HBP in the non-GH group (open bar; $n=785$ ) and the GH group (solid bar; $n=28$ ). Panel a, systolic blood pressure; panel $\mathbf{b}$, diastolic blood pressure. CBP and HBP were analyzed using analysis of variance adjusted for maternal age and body mass index at 7-year survey, education status, parity and current drinking and smoking status. Error bars denote s.d. P-values express the differences between GH and non-GH after adjusting for maternal age and body mass index at 7-year survey, educational status, parity, family history of hypertension, and current drinking and smoking status. $\mathrm{GH}$, gestational hypertension; $\mathrm{CBP}$, conventional blood pressure; HBP, home blood pressure. 


\section{DISCUSSION}

This is the first study investigating the association between GH and self-measured HBP as well as CBP captured 7 years after delivery. In this birth cohort population, maternal GH did not affect BP levels or $\mathrm{BP}$ variability in offspring. However, experience with GH affected the mother's own high HBP levels even when multivariable-adjustment models were applied. We obtained confirmatory results when the initial HBP value was used. The use of self-measured HBP has been reported to have a better reproducibility and prognostic value than CBP in both adults ${ }^{8,24}$ and children. ${ }^{9,10}$ Our findings suggest that measurement not only by CBP but also by self-measured HBP provides more useful prognostic information for women who experienced $\mathrm{GH}$ at an earlier or middle age.

Women with $\mathrm{GH}$ or preeclampsia have an increased risk of cardiovascular disease, ${ }^{25,26}$ cerebrovascular disease ${ }^{23,25}$ and hypertension ${ }^{23}$ in later life. Our results were consistent with previous studies. Mori et al. ${ }^{27}$ reported that $\mathrm{BP}$ in women with preeclampsia

Table 2 Comparison of initial home blood pressure at 7-year survey by $\mathrm{GH}$ categories

\begin{tabular}{|c|c|c|c|}
\hline Variables & Non-GH $(\mathrm{n}=785)$ & $G H(n=28)$ & P-values \\
\hline \multicolumn{4}{|l|}{ Mothers } \\
\hline $\begin{array}{l}\text { Initial home systolic blood pres- } \\
\text { sure, } \mathrm{mmHg}\end{array}$ & $110.1 \pm 12.5$ & $119.2 \pm 14.7$ & $0.006^{a}$ \\
\hline $\begin{array}{l}\text { Initial home diastolic blood } \\
\text { pressure, } \mathrm{mmHg}\end{array}$ & $69.2 \pm 9.7$ & $76.6 \pm 10.7$ & $0.002^{a}$ \\
\hline \multicolumn{4}{|l|}{ Children } \\
\hline $\begin{array}{l}\text { Initial home systolic blood pres- } \\
\text { sure, } \mathrm{mmHg}\end{array}$ & $93.8 \pm 10.0$ & $92.7 \pm 7.8$ & $0.17^{b}$ \\
\hline $\begin{array}{l}\text { Initial home diastolic blood } \\
\text { pressure, } \mathrm{mmHg}\end{array}$ & $56.2 \pm 9.4$ & $56.5 \pm 8.5$ & $0.84^{b}$ \\
\hline
\end{tabular}

Abbreviation: GH, gestational hypertension.

Numbers are shown as mean \pm s.d.

${ }^{a}$ Adjusted by maternal age and body mass index at 7-year survey, educational status, parity family history of hypertension, current drinking and smoking.

${ }^{\text {b} A d j u s t e d ~ b y ~ s e x, ~ b i r t h ~ w e i g h t, ~ a g e ~ a t ~ 7-y e a r ~ s u r v e y, ~ c h i l d r e n ' s ~ b o d y ~ m a s s ~ i n d e x ~ a t ~ 7-y e a r ~}$ survey, parental education status, maternal age at delivery, parity, maternal smoking during pregnancy, maternal body mass index before pregnancy, cesarean section and gestational week. significantly decreased a month after delivery, whereas BP remained higher than in normotensive mothers. Furthermore, nighttime systolic $\mathrm{BP}$ in the ambulatory setting was significantly $(P=0.030)$ elevated in Swedish women with preeclampsia compared with those without preeclampsia even 11 years after delivery, although this was not the case for the other BP data $(P \geqslant 0.073) .{ }^{28}$ According to the guidelines of the International Society for the Study of Hypertension in Pregnancy, $\mathrm{PIH}$ is defined as hypertension with or without proteinuria that resolves within 12 weeks postpartum. ${ }^{12}$ Although we did not collect BP data beyond 12 weeks after delivery, HBP and CBP in the GH women were significantly higher than those in non-GH women even 7 years after giving birth. Our findings support the importance for women who developed hypertension during pregnancy to manage their own health even in middle age or earlier.

The Avon Longitudinal Study of Parents and Children is a large, population-based pregnancy cohort. ${ }^{29,30}$ A total of 13617 mothers with a live singleton birth consented to have their obstetric data abstracted from medical records. The mean (s.d.) age of the eligible 2888 offspring was 208.5 (11.2) months old, and the proportion of preterm delivery before 37 weeks was $4.4 \%{ }^{15}$ CBP in the Avon Longitudinal Study of Parents and Children was significantly higher in 17-year-old offspring of mothers with GH $(120.5 \pm 11.3 / 66.0 \pm 7.2$ $\mathrm{mm} \mathrm{Hg})$ or preeclampsia $(120.2 \pm 10.1 / 66.6 \pm 7.0 \mathrm{~mm} \mathrm{Hg})$ compared with non-PIH $(117.6 \pm 10.4 / 64.5 \pm 6.8 \mathrm{~mm} \mathrm{Hg})$ even when adjusting for potential confounding factors in multivariable linear regression analysis. $^{15}$

Inconsistent with earlier studies, ${ }^{15,31}$ including the Avon Longitudinal Study of Parents and Children, there were no significant associations between maternal GH and HBP in children at 7 years old in the present study. These findings were confirmatory when the 1:2 matched-case analyses were performed. We obtained contradictory findings for systolic CBP $(93.9 \mathrm{~mm} \mathrm{Hg}$ in matched non-GH group $v s$. $90.0 \mathrm{~mm} \mathrm{Hg}$ in GH group), although this opposite result might be due to the lower number of CBP measurements and inaccuracy of BP information compared with home measurements. ${ }^{9}$ In the present study, the rate of children with a preterm birth was $1.6 \%$. This prevalence is lower than the Avon Longitudinal Study of Parents and Children, in which $4.4 \%$ of children were preterm, whereas the inclusion criteria of birth weight in our study was $>2400 \mathrm{~g}$.
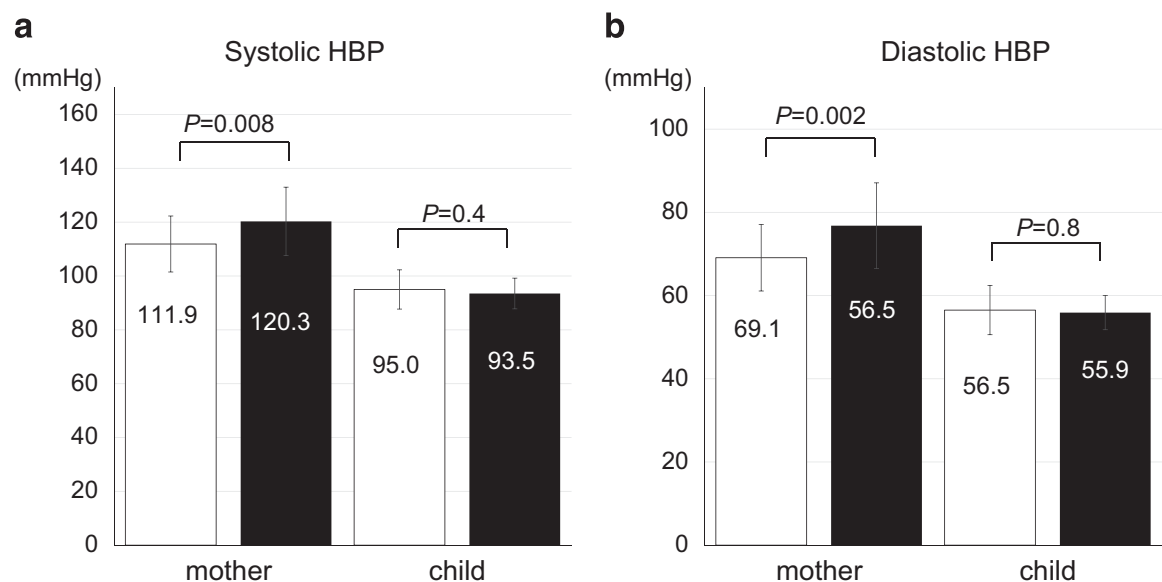

Figure 4 Maternal and children's HBP in the matched non-GH group (open bar; $n=56$ ) and the GH group (solid bar; $n=28$ ). Panel a, systolic HBP; panel $\mathbf{b}$, diastolic HBP. Error bars denote s.d. P-values express the differences between GH and non-GH after adjusting for the following variables: for children, sex, birth weight, age at 7-year survey, body mass index at 7-year survey, parental education status, maternal smoking during pregnancy, cesarean section and gestational week; for mothers, education status and current drinking and smoking status. GH, gestational hypertension; HBP, home blood pressure. 
Table 3 Blood pressure levels at 7-year survey in relation to pregnancy-induced hypertension

\begin{tabular}{|c|c|c|c|}
\hline Variables & Non-PIH $(n=785)$ & $P I H(n=30)$ & Matched non-PIH $(n=60)$ \\
\hline \multicolumn{4}{|l|}{ Mothers } \\
\hline \multicolumn{4}{|l|}{ Conventional measurements } \\
\hline Diastolic blood pressure, $\mathrm{mmHg}$ & $70.5 \pm 9.6^{*}$ & $79.6 \pm 12.4$ & $71.9 \pm 8.5^{*}$ \\
\hline Heart rate, beats $\mathrm{min}^{-1}$ & $68.0 \pm 9.3$ & $68.4 \pm 10.0$ & $68.8 \pm 9.2$ \\
\hline \multicolumn{4}{|l|}{ Home measurements } \\
\hline Diastolic blood pressure, $\mathrm{mmHg}$ & $68.6 \pm 8.3 *$ & $76.5 \pm 10.1$ & $69.7 \pm 6.9 *$ \\
\hline Heart rate, beats $\min ^{-1}$ & $69.2 \pm 7.5$ & $69.9 \pm 7.1$ & $69.7 \pm 6.9$ \\
\hline \multicolumn{4}{|l|}{ Children } \\
\hline \multicolumn{4}{|l|}{ Conventional measurements } \\
\hline Systolic blood pressure, mmHg & $93.1 \pm 9.2$ * & $90.4 \pm 6.5$ & $94.5 \pm 7.9$ * \\
\hline Systolic blood pressure, $\mathrm{mmHg}$ & $94.1 \pm 6.9$ & $93.3 \pm 5.6$ & $95.5 \pm 7.8$ \\
\hline Diastolic blood pressure, $\mathrm{mmHg}$ & $56.1 \pm 5.7$ & $55.7 \pm 4.1$ & $56.7 \pm 6.2$ \\
\hline Heart rate, beats $\mathrm{min}^{-1}$ & $84.1 \pm 7.6$ & $85.3 \pm 7.6$ & $84.4 \pm 7.6$ \\
\hline
\end{tabular}

Preeclampsia at term, accompanied by offspring that appear to be unaffected by the condition, may represent a mixture of conditions, ranging from mild preeclampsia with moderate placental involvement to hypertensive conditions without placental dysfunction. ${ }^{32}$ Offspring born to preeclamptic mothers at term have fetal growth similar to that of babies born to normotensive mothers. ${ }^{33}$ Our study includes fewer preterm births or low-birth-weight infants compared with previous studies, suggesting that maternal GH might have little influence on BP in 7-year-old children who were born at term and had normal birth weight.

The present study must be interpreted with the context of its limitations. First, because our study population consisted of residents from specific Japanese districts, our findings might not be generalizable to other populations. Second, we could not collect mothers' BP before pregnancy and after 12 weeks postpartum. According to the guidelines, the definition of transient hypertension of pregnancy is that maternal CBP returns to normal by 12 weeks postpartum. ${ }^{11,12}$ Some mothers with chronic hypertension might have inadvertently been included in our present analysis. However, we excluded mothers with severe complications at the entry period and those who were under antihypertensive drug medication at the 7-year survey. We may have included mothers with white-coat hypertension in the GH group because we did not measure maternal HBP during pregnancy. Third, we could not assess GH and preeclampsia separately because of the limited number of preeclamptic women $(n=2)$, and there were only 30 women with PIH in our population. We might have overlooked small differences in children's BP between GH and non-GH because it may reflect a beta error due to the comparably small number of women with GH. Further studies with large sample sizes will be required to confirm the associations of maternal GH and preeclampsia with HBP in children and whether the differences in population characteristics (for example, birth weight, rate of preterm) affect these associations. Finally, our findings might not be applicable to preterm and low-birth-weight children born to PIH mothers who exhibit preterm birth and intrauterine growth restriction. ${ }^{34,35}$ Because our study population consists primarily of term children, our results could be applied to term children born to PIH mothers.

In conclusion, maternal GH did not affect HBP in offspring with normal birth weight and at term but strongly affected maternal HBP even 7 years after giving birth. Preventive strategies focusing on PIH may lead to lowering PIH women's morbidity and mortality of cardiovascular disease in the future. Our findings support the importance for women who experienced hypertensive disorders during pregnancy to manage their own health even in middle age or earlier and indicate that growth environment has an important influence on HBP in children.

\section{CONFLICT OF INTEREST}

The authors declare no conflict of interest.

\section{ACKNOWLEDGEMENTS}

We gratefully acknowledge all the staff members of the Tohoku Study of Child Development for their valuable contributions to data collection and database management. We also thank all the participants in the study. Sources of funding: the study was supported by Grants for Scientific Research (23390171) from the Ministry of Education, Culture, Sports, Science and Technology, Japan; a Grant-in-Aid for Japan Society for the Promotion of Science (JSPS) fellows (25.7756 and 25.9328); the Ministry of Health, Labour and Welfare, Japan; the Ministry of the Environment, Japan; and the Grant for Environmental Research Projects from the Sumitomo Foundation. 
1 Ohkubo T, Imai Y, Tsuji I, Nagai K, Watanabe N, Minami N, Itoh O, Bando T, Sakuma M, Fukao A, Satoh H, Hisamichi S, Abe K. Prediction of mortality by ambulatory blood pressure monitoring versus screening blood pressure measurements: a pilot study in Ohasama. J Hypertens 1997: 15: 357-364.

2 Lawes CM, Rodgers A, Bennett DA, Parag V, Suh I, Ueshima H, MacMahon S, Asia Pacific Cohort Studies Collaboration. Blood pressure and cardiovascular disease in the Asia Pacific region. J Hypertens 2003; 21: 707-716.

3 Lida M, Ueda K, Okayama A, Kodama K, Sawai K, Shibata S, Tanaka S, Keijnkai T, Horibe H, Minowa M, Yanagawa H, Hashimoto T, Nippon Data 80 Research Group. Impact of elevated blood pressure on mortality from all causes, cardiovascular diseases, heart disease and stroke among Japanese: 14 year follow-up of randomly selected population from Japanese - Nippon data 80. J Hum Hypertens 2003; 17: 851-857.

4 Asayama K, Ohkubo T, Yoshida S, Suzuki K, Metoki H, Harada A, Murakami Y, Ohashi $Y$, Ueshima H, Imai $Y$, the Japan Arteriosclerosis Longitudinal Study (JALS) group. Stroke risk and antihypertensive drug treatment in the general population: the Japan arteriosclerosis longitudinal study. J Hypertens 2009; 27: 357-364.

5 Kollias A, Pantsiotou K, Karpettas N, Roussias L, Stergiou GS. Tracking of blood pressure from childhood to adolescence in a Greek cohort. Eur J Public Health 2012. 22: 389-393.

6 Sun SS, Grave GD, Siervogel RM, Pickoff AA, Arslanian SS, Daniels SR. Systolic blood pressure in childhood predicts hypertension and metabolic syndrome later in life. Pediatrics 2007; 119: 237-246.

7 Ohkubo T, Imai Y, Tsuji I, Nagai K, Kato J, Kikuchi N, Nishiyama A, Aihara A, Sekino M, Kikuya M, Ito S, Satoh H, Hisamichi S. Home blood pressure measurement has a stronger predictive power for mortality than does screening blood pressure measurement: a population-based observation in Ohasama, Japan. J Hypertens 1998; 16: 971-975.

8 Ohkubo T, Asayama K, Kikuya M, Metoki H, Hoshi H, Hashimoto J, Totsune K, Satoh H, Imai Y, Ohasama S. How many times should blood pressure be measured at home for better prediction of stroke risk? Ten-year follow-up results from the Ohasama study. J Hypertens 2004; 22: 1099-1104.

9 Stergiou GS, Nasothimiou EG, Giovas PP, Rarra VC. Long-term reproducibility of home vs office blood pressure in children and adolescents: the Arsakeion school study. Hypertens Res 2009; 32: 311-315.

10 Stergiou GS, Giovas PP, Kollias A, Rarra VC, Papagiannis J, Georgakopoulos D, Vazeou A. Relationship of home blood pressure with target-organ damage in children and adolescents. Hypertens Res 2011; 34: 640-644.

11 Japan Society for Study of Hypertension In Pregnancy. Guideline 2009 for care and treatment of hypertension in pregnancy (PIH). Medical View Co., Ltd., 2009. (in Japanese).

12 Brown MA, Lindheimer MD, de Swiet M, Van Assche A, Moutquin JM. The classification and diagnosis of the hypertensive disorders of pregnancy: statement from the International Society for the Study of Hypertension in Pregnancy (ISSHP). Hypertens Pregnancy 2001; 20: IX-XIV.

13 Sibai B, Dekker G, Kupferminc M. Pre-eclampsia. Lancet 2005; 365: 785-799.

14 Geelhoed JJ, Fraser A, Tilling K, Benfield L, Davey Smith G, Sattar N, Nelson SM, Lawlor DA. Preeclampsia and gestational hypertension are associated with childhood blood pressure independently of family adiposity measures: the Avon Longitudinal Study of Parents and Children. Circulation 2010; 122: 1192-1199.

15 Fraser A, Nelson SM, Macdonald-Wallis C, Sattar N, Lawlor DA. Hypertensive disorders of pregnancy and cardiometabolic health in adolescent offspring. Hypertension 2013, 62: 614-620.

16 Tenhola S, Rahiala E, Halonen P, Vanninen E, Voutilainen R. Maternal preeclampsia predicts elevated blood pressure in 12-year-old children: evaluation by ambulatory blood pressure monitoring. Pediatr Res 2006; 59: 320-324.

17 Vatten L. Intrauterine exposure to preeclampsia and adolescent blood pressure, body size, and age at menarche in female offspring. Obstet Gynecol 2003; 101: 529-533.
18 Ferreira I, Peeters LL, Stehouwer CD. Preeclampsia and increased blood pressure in the offspring: meta-analysis and critical review of the evidence. J Hypertens 2009; 27 1955-1959.

19 Nakai K, Suzuki K, Oka T, Murata K, Sakamoto M, Okamura K, Hosokawa T, Sakai T, Nakamura T, Saito Y, Kurokawa N, Kameo S, Satoh H. The Tohoku Study of Child Development: a cohort study of effects of perinatal exposures to methylmercury and environmentally persistent organic pollutants on neurobehavioral development in Japanese children. Tohoku J Exp Med 2004; 202: 227-237.

20 Asayama K, Staessen JA, Hayashi K, Hosaka M, Tatsuta N, Kurokawa N, Satoh M, Hashimoto T, Hirose T, Obara T, Metoki H, Inoue R, Kikuya M, Ohkubo T, Nakai K, Imai Y, Satoh H. Mother-offspring aggregation in home versus conventional blood pressure in the Tohoku Study of Child Development (TSCD). Acta Cardiol 2012; 67: 449-456.

21 North RA, Taylor RS, Schellenberg JC. Evaluation of a definition of pre-eclampsia Br J Obstet Gynaecol 1999; 106: 767-773.

22 Stergiou GS, Yiannes NG, Rarra VC. Validation of the Omron 705 IT oscillometric device for home blood pressure measurement in children and adolescents: the Arsakion School Study. Blood Press Monit 2006; 11: 229-234.

23 Wilson BJ, Watson MS, Prescott GJ, Sunderland S, Campbell DM, Hannaford P, Smith WC. Hypertensive diseases of pregnancy and risk of hypertension and stroke in later life: results from cohort study. BMJ 2003; 326: 845.

24 Imai Y, Obara T, Asamaya K, Ohkubo T. The reason why home blood pressure measurements are preferred over clinic or ambulatory blood pressure in Japan. Hypertens Res 2013; 36: 661-672.

25 Mannisto T, Mendola P, Vaarasmaki M, Jarvelin MR, Hartikainen AL, Pouta A, Suvanto E. Elevated blood pressure in pregnancy and subsequent chronic disease risk. Circulation 2013; 127: 681-690.

26 Irgens HU, Reisaeter L, Irgens LM, Lie RT. Long term mortality of mothers and fathers after pre-eclampsia: population based cohort study. BMJ 2001; 323: 1213-1217.

27 Mori T, Watanabe K, Iwasaki A, Kimura C, Matsushita H, Shinohara K, Wakatsuki A. Differences in vascular reactivity between pregnant women with chronic hypertension and preeclampsia. Hypertens Res 2014; 37: 145-150.

28 Ostlund E, Al-Nashi M, Hamad RR, Larsson A, Eriksson M, Bremme K, Kahan T. Normalized endothelial function but sustained cardiovascular risk profile 11 years following a pregnancy complicated by preeclampsia. Hypertens Res 2013; 36: 1081-1087.

29 Fraser A, Macdonald-Wallis C, Tilling K, Boyd A, Golding J, Davey Smith G, Henderson J, Macleod J, Molloy L, Ness A, Ring S, Nelson SM, Lawlor DA. Cohort Profile: the Avon Longitudinal Study of Parents and Children: ALSPAC mothers cohort. Int J Epidemiol 2013; 42: 97-110.

30 Boyd A, Golding J, Macleod J, Lawlor DA, Fraser A, Henderson J, Molloy L, Ness A Ring S, Davey Smith G. Cohort Profile: the 'children of the 90s'-the index offspring of the Avon Longitudinal Study of Parents and Children. Int J Epidemiol 2013; 42 111-127.

31 Mamun AA, Kinarivala MK, O'Callaghan M, Williams G, Najman J, Callaway L. Does hypertensive disorder of pregnancy predict offspring blood pressure at 21 years? Evidence from a birth cohort study. J Hum Hypertens 2012; 26: 288-294.

32 Vatten LJ, Skjaerven R. Is pre-eclampsia more than one disease? BJOG 2004; 111 298-302.

33 Xiong X, Demianczuk NN, Saunders LD, Wang FL, Fraser WD. Impact of preeclampsia and gestational hypertension on birth weight by gestational age. Am J Epidemiol 2002; 155: 203-209.

34 Ferrazzani S, Luciano R, Garofalo S, D'Andrea V, De Carolis S, De Carolis MP, Paolucci V. Romagnoli C, Caruso A. Neonatal outcome in hypertensive disorders of pregnancy. Early Hum Dev 2011; 87: 445-449.

35 Xiong X, Demianczuk NN, Buekens P, Saunders LD. Association of preeclampsia with high birth weight for age. Am J Obstet Gynecol 2000; 183: 148-155. 